\title{
O uso de indicadores de infecção em Unidade de Terapia Intensiva: uma revisão da literatura
}

\author{
The use of infection indicators in the Intensive Care Unit: a literature review \\ El uso de indicadores de infección en la Unidad de Cuidados Intensivos: revisión de la \\ literatura
}

Daniel Ghiraldelli*, Gabriela Moretto do Nascimento, Felipe Neri Nunes Venâncio², Sarah Russo Calil1, Aline Bedin Zanatta1, Cristiane Pereira de Castro1, Luis Eduardo Miani Gomes¹.

\section{RESUMO}

Objetivo: Descrever os indicadores empregados na Unidade de Terapia Intensiva, identificando a utilidade e aplicação, e os obstáculos. Revisão bibliográfica: A literatura corrobora e adverte o uso de ferramentas para o aparelhamento da assistência de enfermagem apoiado na necessidade de promover e viabilizar o processo educativo entre os enfermeiros, pela própria atribuição que lhe pertence. Nota-se que as infecções hospitalares avançam no tempo, e as interferências devem ser estabelecidos. A Unidade de Terapia Intensiva é responsável por grande parte das infecções contraídas. Desse modo, os indicadores transportam a uma direção e orientação possibilitando assim que a equipe multiprofissional possa adotar atitudes preventivas e reparativas na assistência ao paciente. Considerações finais: Os indicadores da Unidade de Terapia Intensiva têm-se demonstrado de grande aplicabilidade e aproveitamento, aliado às práticas assistências dos profissionais de saúde, em especial dos enfermeiros, que dentro dessa égide deve conhecer e orientar-se sobre o objeto (indicador) a ser empregado, bem como os meios e os resultados encontrados nessa investigação.

Palavras-chave: Indicadores, Unidade de terapia intensiva, Infecção hospitalar.

\begin{abstract}
Objective: To describe the indicators used in the Intensive Care Unit, identifying the utility and application, and the obstacles. Bibliographic review: The literature corroborates and warns the use of tools for equipping nursing care based on the need to promote and facilitate the educational process among nurses, due to the assignment that belongs to them. It is noted that hospital infections advance over time, and interferences must be established. The Intensive Care Unit is responsible for most of the infections contracted. In this way, the indicators convey direction and guidance, thus enabling the multidisciplinary team to adopt preventive and reparative attitudes in patient care. Final considerations: The indicators of the Intensive Care Unit have proven to be of great applicability and use, combined with the practical assistance of health professionals, especially nurses, who within this aegis must know and orient themselves about the object (indicator) to be employed, as well as the means and results found in that investigation.
\end{abstract}

Key words: Indicators, Intensive care unit, Cross infection.

\section{RESUMEN}

Objetivo: Describir los indicadores utilizados en la Unidad de Cuidados Intensivos, identificando la utilidad y aplicación, y los obstáculos. Revisión bibliográfica: La literatura corrobora y advierte el uso de herramientas para equipar el cuidado de enfermería en base a la necesidad de promover y facilitar el proceso educativo entre los enfermeros, debido a la asignación que les corresponde. Se observa que las infecciones hospitalarias avanzan con el tiempo y se deben establecer interferencias. La Unidad de Cuidados Intensivos es responsable de la mayoría de las infecciones contraídas. De esta forma, los indicadores transmiten dirección y orientación, lo que permite que el equipo multidisciplinar adopte actitudes preventivas y

\footnotetext{
${ }^{1}$ Faculdade de Americana (FAM), Americana - SP. *E-mail: fisio.danielghiraldelli@hotmail.com

2 Irmandade de Misericórdia de Campinas - Hospital Irmãos Penteado, Campinas - SP.
} 
reparadoras en la atención al paciente. Consideraciones finales: Los indicadores de la Unidad de Cuidados Intensivos han demostrado ser de gran aplicabilidad y utilidad, sumado a las prácticas asistenciales de los profesionales de la salud, especialmente enfermeras, quienes dentro de esta égida deben conocer y orientarse sobre el objeto (indicador) a emplear, así como los medios y resultados encontrados en dicha investigación.

Palabras clave: Indicadores, Unidad de cuidados intensivos, Infección hospitalaria.

\section{INTRODUÇÃO}

No Brasil o número relacionado às infecções hospitalares vem crescendo nos últimos anos, tendo maiores incidências em rede pública de saúde, sendo a Unidade de Terapia Intensiva (UTI) de maior ocorrência devido ao grau de complexidade de cada paciente, possuindo alto risco para contrair uma infecção hospitalar. A UTI juntamente com os profissionais envolvidos tem grande importância no desempenho da sobrevida dos pacientes internados. A infecção hospitalar não é apenas uma complicação evitável em uma UTI, porém está intimamente relacionada também a possibilidades de melhorias na qualidade de assistência que vem sendo prestada (FIGUEIREDO DA, et al., 2013).

Segundo o manual da Joint Comission Acreditation of Healthcare Organizations (JCAHO), indicadores tem como função primordial avaliar o desempenho de funções, a meio de organizar e detalhar a direção do processo e estipular uma meta a longo prazo. Os indicadores na enfermagem como um todo se fazem necessários, não apenas como uma tarefa na assistência, mas algo que faça parte de todo o processo de enfermagem, para que os resultados finais sejam alcançados de maneira positiva, resultando na qualificação e melhorias no atendimento dos serviços hospitalares (CALDANA G, et al., 2011).

As infecções hospitalares são epidemiologicamente pertinentes, pois aumentam as taxas de morbimortalidade, ampliando o tempo de permanência dos pacientes no ambiente hospitalar, e consequentemente aumentando os custos de sua internação e tratamentos. O setor da Unidade de Terapia Intensiva é responsável por quase a metade das infecções hospitalares adquiridas, uma média de 35 a $45 \%$, sendo a causa mais comum a nosocomial, acarretando um prolongamento nas internações, gerando altos custos para a instituição (MENEGUETTI MG, et al., 2012).

No ambiente hospitalar o uso dos indicadores vem crescendo nos últimos anos, sendo uma forma quantitativa, periódica, que através dos dados coletados revelam se os processos obtiveram resultados desejáveis ou indesejáveis, ou seja, possibilitam através de critérios objetivar a qualidade dos serviços de saúde, podendo um indicador mostrar a qualidade do serviço prestado ou algum comprometimento, que possa diretamente afetar à assistência (MENEGUETTI MG, et al., 2012).

A aplicação dos indicadores na UTI é rotina para muitos profissionais. A UTI é um lugar proposto a assistir pacientes graves e instáveis que, comumente, é considerado de alta complexidade, por conta do aparato tecnológico e informatizado de ponta, que oferece ritmo acelerado, no qual são materializados procedimentos agressivos e invasivos, que necessitam ser monitorados 24 horas, onde o duelo entre a vida e a morte está bem presente, sendo que a morte, continuamente, é iminente (BACKES MTS, et al., 2015).

Destaca-se que os membros dessa equipe multiprofissional são formados por enfermeiros, técnicos de enfermagem, médicos, fisioterapeutas, nutricionistas, psicólogos dentre outros (ABRAHÃO ALCL, 2011). A UTI é um local marcado pela prestação de cuidados por profissionais conhecedores das atividades especializadas destinadas a pacientes graves (SALOMÉ M, et al., 2010).

O uso de ferramentas para organização da assistência de enfermagem bem como a necessidade de promover e viabilizar o processo educativo entre os enfermeiros, impulsiona esses profissionais a cada vez mais utilizar métodos técnicos e científicos recomendados (MENDES NR, et al., 2019).

Outrossim, uma equipe qualificada e preparada técnica e assistencialmente para desenvolver as habilidades relacionadas às suas competências, apropriadamente aos pacientes internados na UTI, tem-se 
uma grande redução das taxas de incidência das infecções, por exemplo. Não menos importante, no processo de indicadores e levantamento de dados e informações à Comissão de Controle de Infecção Hospitalar (CCIH) está diretamente ligada, claro que juntamente com o enfermeiro responsável pela unidade. Assim sendo, o trabalho em equipe (enfermeiros e $\mathrm{CCIH}$ ) pode proporcionar aos pacientes atitudes preventivas de infecções, e consequente menor risco de infecção durante a permanência na UTI (FRANÇA LM, et al., 2019).

Diante dessas perspectivas, observa-se a necessidade de entender e estender os conhecimentos à cerca dos indicadores usados na UTI de forma a analisar o ambiente, e as dificuldades enfrentadas. Assim, o presente artigo tem como objetivo descrever indicadores utilizados na Unidade de Terapia Intensiva, identificando a aplicabilidade, e os obstáculos para sua execução.

\section{REVISÃO BIBLIOGRÁFICA}

Para a realização do presente estudo foi realizado a busca por referências sobre a temática através das palavras-chave, que ocorreu de Novembro de 2020 a Fevereiro de 2021, incluindo artigos dos últimos 10 anos, através das bases de dados das ciências da saúde. Os critérios de exclusão foram: artigos incompletos, resumos de congresso e artigos que não respondessem à temática e/ou não descreviam o conteúdo de forma a contribuir com o presente estudo. Um novo conceito relacionado às Infecções Hospitalares vem ganhando força, não restringindo apenas ao ambiente hospitalar, podendo sim, ocorrer em demais setores, seja unidade básica de saúde, serviços como Home Care e também no ambiente domiciliar (FERNANDES ACL, et al., 2014).

Dentro de uma UTI a assistência é realizada de forma especializada, particularizada, onde os pacientes recebem cuidados e assistência permanente. Ambiente de grande complexidade relacionado a recurso de última geração tecnológica, sendo necessários para o monitoramento contínuo (FERNANDES ACL, et al., 2014).

Em face da ampliação do ambiente hospitalar, e mediante as diversificações das unidades de terapia intensiva, a aplicação dos indicadores deve ser personalizada e observar as características hospitalares, setoriais, produtivas e quantitativas de funcionários da área. Os erros relacionados aos cuidados sempre estiveram presentes em toda a trajetória da história da saúde, porém desde os princípios, já se pensava em melhorias no cuidado e segurança do paciente (VIEIRA MAN e BERETTA ALRZ, 2018).

Ao longo dos anos a evolução científica vem se fazendo presente, resultando em ações positivas prestadas aos pacientes. Mas juntamente com a evolução notam-se problemas antigos e que ainda persistem como as Infecções Hospitalares, sendo os pacientes internados em uma unidade de terapia intensiva mais susceptível e vulnerável aos agravos (VIEIRA MAN e BERETTA ALRZ, 2018).

As infecções hospitalares obtiveram grandes aumentos nos últimos 20 anos devido à quantidade de procedimentos invasivos, a resistência bacteriana a antibióticos que se fazem ineficazes no tratamento, e também as falhas no controle de infecções. Estudos realizados em 2010 relatam que em 2005 os números de infecções hospitalares chegaram a 800 mil casos (FIGUEIREDO AD, 2012).

Seguindo a linha de descrições a UTI é usada para proporcionar cuidados diretos ao paciente que precisam de assistência médica e de enfermagem, acautelando a evolução do estado clínico, impedindo o comprometimento da vida (MACHADO EA, 2004). Nas atribuições e competências dos enfermeiros, a Sistematização da Assistência de Enfermagem (SAE), cabe ao enfermeiro às condutas de planejar, analisar e implementar as ações, oportunizando atendimento individualizado ao paciente com qualidade (ZANARDO GM, et al., 2011).

Noutro estudo há relatos da importância dos dados coletados pela CCIH. A CCIH era formada por um médico, duas enfermeiras capacitadas no controle de infecções e apoio dos profissionais da área de estatística, que visam descrever as infecções hospitalares, o que elas acarretam na qualidade da assistência, os custos relacionados e contribuir com melhorias, evitando e também reduzindo os casos de infecções. Esses dados são coletados a partir de buscas intensivas e rotineiras de casos de infecções a busca de casos 
juntamente com os denominadores para os cálculos de indicadores são realizados pelas enfermeiras responsáveis (PRATES DB, et al., 2014).

Em que pese os fundamentos, nota-se a necessidade do controle das infecções hospitalares, em especial a UTI, sendo de suma importância estratégias no controle e redução aos números de infecções relacionadas às internações e aos procedimentos realizados dentro do ambiente hospitalar. Os pacientes suscetíveis internados em uma UTI, independente do seu quadro, estão mais sujeitos a adquirir não apenas a infecções, mas sua evolução para uma sepse, e consequentemente comprometendo a continuidade à vida. Pacientes internados em UTI possuem uma probabilidade de 5 a 10 vezes mais de contrair uma infecção (REINER GL, et al., 2020).

\section{Os indicadores: objeto, Meio e Resultado. O Objeto}

Dentro do ambiente hospitalar as taxas de infecções são fatores primordiais para proporcionar a segurança ao paciente, e consequentemente resultando em melhorias assistenciais. Através dos indicadores, buscamse medidas de controle precoce, sendo uma ferramenta fundamental nos serviços de saúde, de modo a contribuir no decrescimento de infecções. Porém, destaca-se a necessidade dos profissionais de saúde envolvidos, conhecer e demonstrar os resultados obtidos dos indicadores, para toda a equipe, a fim de contribuir de forma positiva nos programas de controle e prevenção (SILVEIRA JS, 2019).

A implantação de indicadores, em especial na UTI é um método vastamente utilizado na prática dos profissionais de saúde, em especial aos enfermeiros. Pela própria atribuição que the compete de acordo com o Código de Ética em Enfermagem, os princípios fundamentais de sua atribuição é a de assistir, gerenciar, ensinar, educar e pesquisar (CONSELHO FEDERAL DE ENFERMAGEM, 2017). Nesses princípios basilares, os indicadores são inseridos de forma direta e indireta de forma a buscar uma assistência que possibilite um cuidado profissional seguro e livre de danos.

Os indicadores servem para direcionar e orientar um caminho ou possibilidades de caminhos, pois auxiliam a pensar atitudes de conduzir tendo em vista pontos socioambientais e econômicas (REIS ML, et al., 2011). No mesmo sentido, podemos destacar que nessa prática assistencial, os indicadores também estão relacionados não somente com o serviço assistencial como também relacionado aos índices de morbimortalidade (SANTANA TCP, et al., 2019).

Vale ressaltar que os pacientes internados dentro de uma instituição de saúde, em especial à UTI, estão sujeitos a contaminação por uma vasta variedade de microrganismos, comparado com os demais setores dentro da instituição, devido sua vulnerabilidade e aos procedimentos invasivos de rotina e o uso elevado de antimicrobianos, fazendo com que esses microrganismos se tornem mais resistentes a terapia. Os pacientes em uma UTI têm maior probabilidade de uma Infecção Hospitalar devido sua complexidade, ou seja, fragilidade após uma cirurgia complexa, baixa imunidade, vários profissionais envolvidos e o uso de antimicrobianos (ABRGG PTGM e SILVA LL, 2011).

Os indicadores podem ser introduzidos e estabelecidos na prática assistencial dos profissionais de enfermagem. Além disso, a empregabilidade e a eficácia dos indicadores devem ser robustamente testadas empregando a rigidez e precisão técnica científica presente, bem como um método coerente e acessível (MAINZ J, 2003).

Os indicadores dentro do programa de controle de infecção devem estar presentes de forma diária prioritária em uma Unidade de terapia Intensiva, de modo que seja observado detalhadamente a vigilância de Infecções Relacionadas à Assistência à Saúde (IRAS), sendo muito frequente em pacientes internados em uma UTI, vulneráveis a infecções, podendo trazer vários desfechos negativos. O controle de infecção deve ser realizado de forma sistemática, por todos envolvidos no processo, resultando no restabelecimento da qualidade da assistência. Vale ressaltar a importância da qualificação dos profissionais para que o resultado esperado seja positivo, e os cuidados assertivos quanto aos pacientes sépticos e que devem ter cuidados imediatos, pois estudos demonstram altos índices de mortalidade em pacientes com choque séptico dentro das Unidades de Terapia Intensiva (RODRIGUES CN e PEREIRA DC, 2016). 


\section{O Meio}

Dentro da esfera hospitalar podemos apartar que o controle de alguns indicadores é feito pela $\mathrm{CCIH}$ tem atuação diretamente com a equipe multidisciplinar, e que de acordo com as normas vigentes, é responsável pela vigilância das infecções, apresentando os indicadores recomendados pela Agência Nacional de Vigilância Sanitária (ANVISA) àqueles apresentados nos indicadores nacionais de infecções relacionados à assistência à saúde. Este serviço, dentre outras atribuições realiza observação ativa, sistemática e contínua da ocorrência e da distribuição das infecções entre os pacientes; emprega antibiograma; acompanha as prescrições de antimicrobiano e habilita e prepara os profissionais quão intensamente ao controle e prevenção (BRASIL, 1998).

Levando em conta o impacto por traz das infecções hospitalares, é primordial que os hospitais instituam a $\mathrm{CCIH}$, conforme estabelece a legislação brasileira referente à Portaria de n. $2616 / 98$, referente à implantação e execução do Programa de infecção Hospitalar, com intuito na redução dos índices de infecção à um número mais aceitável, transformando a assistência mais qualificada com excelência e o envolvimento quanto a segurança do paciente. E para que a qualidade da assistência venha à ser mensurada, observa-se à importância da aplicação dos indicadores de avaliação, sendo uma ferramenta para a evolução de melhorias (GIROTI ALB, et al., 2018).

De acordo com a postagem do Ministério da Saúde referente à portaria n. 2616/98 dispõe sobre diretrizes e normas, sendo um dos tópicos a composição da $\mathrm{CCIH}$, em que os membros executores serão, no mínimo, dois técnicos de nível superior da área de saúde para cada 200 leitos, trabalhando em conjunto na $\mathrm{CCIH}$, através de ações estabelecidas na redução das incidências e da gravidade das infecções através do PCIH (BRASIL, 2013).

Outrossim, há ainda relatos de que os microrganismos mais envolvidos nas infecções hospitalares são as Pseudômonas aeruginosa, Acinetobacter baumanni e Klebisiella pneumoniae. As taxas relacionadas a infecções dependerão do nível de complexidade do paciente em uma UTI, do sistema de vigilância e CCHI, ressaltando que a maioria desses pacientes internados são idosos, vulneráveis, devido ao seu próprio processo natural de envelhecimento, tornando-se susceptíveis em internações a longo período, procedimentos invasivos e tratamentos farmacoterapêuticos. Os procedimentos invasivos se fazem presentes em indicadores em UTI, pois quando associados a longo período de permanência, deixam os pacientes mais susceptíveis, sendo uma porta de entrada a infecções. Várias infecções vêm sendo notificadas em pacientes internados em uma UTI como a pneumonia, septicemia e infecções do trato urinário (FIGUEIREDO DA, et al., 2013).

As unidades hospitalares possuem protocolos de atendimento e prevenção de infecção hospitalar, que naturalmente devem estar ativamente em funcionamento, são os indicadores, ferramentas que identificam a não incorporação das medidas preventivas já estabelecidas (MENEGUETI MG, et al., 2012).

A compreensão dos indicadores na prática assistencial, a importância e o impacto da assistência de enfermagem subsidiam diretamente a tomada de decisão, com vistas a excelência do cuidado oferecido (GARCIA PC e FUGULIN FMT, 2012). As decisões para a busca de um resultado partem de medidas administrativas às assistenciais, planejando e delineando um único resultado, que deve ser a melhor assistência ao paciente, livre de negligência, imprudência e imperícia.

Entretanto, a escolha adequada dos indicadores deve ser observada com certa ponderação e, sobretudo para o objeto da qual será empregado. Deve ser empregado na avaliação da qualidade do atendimento, direta e indiretamente e o resultado desses indicadores aos consumidores (profissionais da saúde) ser apresentado (MAINZ J, 2003).

Através da vigilância epidemiológica e indicadores epidemiológicos das infecções hospitalares de forma geral e posteriormente detalhada, tem como visão a aplicação de ações direcionadas a prevenções e controle das contaminações. Dentre este achado podemos citar alguns dos indicadores mais utilizados dentro do contexto hospitalar de forma geral, empregados periodicamente em serviços com maior complexidade (BRASIL, 1998). 
Sendo o Berçário de alto risco, UTI (adulto, pediátrica, neonatal) e queimados sendo eles: Taxa de Infecção Hospitalar; Taxa de Pacientes com Infecção Hospitalar; Distribuição Percentual das Infecções Hospitalares por localização topográfica no paciente; Taxa de Infecções Hospitalares por Procedimento; Frequência das Infecções Hospitalares por microrganismos ou por etiologias; Coeficiente de Sensibilidade aos Antimicrobianos; Indicadores de uso de antimicrobianos; Taxa de Letalidade associada a infecção hospitalar (BRASIL, 1998).

Em relação às Taxas de Infecções Hospitalares por procedimentos, está relacionado ao número de pacientes que foram realizados procedimentos de risco, que consequentemente desenvolveram infecções e o total de pacientes que realizaram esse tipo de procedimento, sendo subdividido em Taxa de Infecção do Sítio Cirúrgico; Taxa de Infecção após Cateterismo Vesical; Taxa de Pneumonia após uso do ventilador mecânico, entre outros (BRASIL, 1998).

\section{O Resultado}

Quando se fala em indicadores, uns dos mais retratados e empregados pelas instituições até mesmo pelos aspectos legais existentes, são os indicadores de infecção hospitalar. Segundo a portaria no 2.616 de 12 de maio de 1998, apresentada pelo Ministério da Saúde (MS), a infecção hospitalar é aquela sendo adquirida após a admissão do paciente, e não a adquirida antes, se manifestando durante a internação ou até mesmo após a alta, sendo a infecção relacionada com a internação ou procedimentos assistenciais (BRASIL, 1998).

Garcia PC e Fugulin FMT (2012) descrevem alguns indicadores utilizados em uma unidade de terapia intensiva adulto, quais são: Incidência de extubação acidental, incidência de perda de sonda nasogástrica e nasoenteral, incidência de perda de cateter venoso central e incidência de úlcera por pressão (GARCIA PC e FUGULIN FMT, 2012).

Esse indicador é comparativo ao tempo de assistência de Enfermagem aplicado por enfermeiros e o indicador de qualidade extubação acidental, da qual se observou que a ocorrência de extubação acidental diminui na proporção que aumentaram as horas de assistência de enfermagem (GARCIA PC e FUGULIN FMT, 2012).

A infecção hospitalar encontrada nas terapias intensivas e, alguns estudos têm demonstrado a incidência de $28,44 \%$ de infecção hospitalar, sendo $22,11 \%$ infecções relacionadas à pneumonia associada ao ventilador mecânico e 6,34\% são infecções do trato urinário (ITU) associadas à Sonda Vesical de Demora (SVD) (FRANÇA FR, et al., 2020).

No estudo de Garcia PC e Fugulin FMT (2012) descrevem, entretanto que no indicador de perda de sonda nasogastroenteral, perda de cateter venoso central e úlcera por pressão não foram observadas correlações estatísticas. Assim, nota-se que nem sempre os indicadores apresentam resultados que eram expectáveis por aqueles que avaliam os dados.

Outro indicador de prevalência descrito é o de infecção urinária, e, seus processos de prevenção. Nota-se que as medidas de prevenção e controle de infecção associado ao cateter vesical estão divulgadas nos protocolos do Centers for Disease Control (CDC) e estão reunidos na ordem de indicação de sondagem, inserção do cateter, melhoramento da condição na inserção, conservação, infraestrutura administrativa e mecanismos de vigilância (MENEGUETI MG, et al., 2012).

Em outro recente estudo, observou-se a prevalência de bactérias Gram-negativas e Gram-positivas em hemoculturas de infecções primárias da corrente sanguínea, onde se identificou que as infecções eram em cateter duplo lúmen em evidência a inserção na veia jugular. Constatou-se que a infecções primárias da corrente sanguínea eram maiores em pacientes que seguiram internados por mais de 15 dias com o referido dispositivo e que presumivelmente estava relacionado com os cuidados na manutenção deste dispositivo (RECH NLM, et al., 2019).

As infecções da corrente sanguínea apresentam vários fatores e fisiopatologia, bem como os critérios diagnósticos. As implicações de uma terapia eficaz, o prognóstico e as medidas preventivas são medidas distintas que devem ser adotadas. As infecções relacionadas ao acesso vascular (IAV) acontecem no sítio de inserção do cateter, não apresentando repercussão sistêmica (BRASIL, 2009). 
No mesmo sentido a ANVISA em 2010 produziu o manual de indicadores de infecções relacionados às principais síndromes infecciosas atinentes a assistência à saúde. Neste, inclui-se as definições, indicadores, medidas e estratégias de prevenção. Destaca-se que o principal objetivo destas recomendações é a definição de indicadores de infecções da qual serão objetos de monitoramento pelas Instituições de saúde (BRASIL, 2010).

As infecções hospitalares estão relacionadas não apenas a complicação diária em pacientes críticos em uma UTI, mas sim como um indicador para melhorias na qualidade assistencial, indicando aos profissionais de saúde envolvidos, uma gama de possibilidades de modificar e/ou qualificarem as medidas preventivas que vem sendo implantadas, com grande intuito na redução de infecções e uma assistência mais segura aos pacientes, diminuindo os riscos e o tempo de internação (FIGUEIREDO DA, et al., 2013).

Tendo em vista a complexidade dentro de uma UTI e dos pacientes que necessitam desses cuidados, não se pode esquecer à importância da enfermagem quanto às intervenções e prevenções, tendo então papel relevante às IRAS. A Sistematização da Assistência de Enfermagem é uma ferramenta de forma legal de extrema importância para a realização da identificação das necessidades, gerenciando os cuidados necessários e atendendo de forma individual, contribuindo para o seguimento assistencial (FERNANDES ACL, et al., 2014).

\section{CONSIDERAÇÕES FINAIS}

Pela literatura estudada, foi destacado, direta ou indiretamente, nesse contexto, os indicadores usados na Unidade de Terapia Intensiva sejam eles, assistências ou não. Tem-se demonstrado sua aplicabilidade nas correções e alinhamentos das práticas assistências dos profissionais de saúde, em especial dos enfermeiros que pela própria formação acadêmica acaba assumindo papel muito importante dentro das unidades críticas, além claro, de outras. De toda forma os enfermeiros devem conhecer e inteirar-se do objeto (indicador) a ser empregado, bem como o meio e os resultados encontrados nessa investigação a fim de instituir medidas corretivas, preventivas e paliativas, possibilitando melhorias na qualidade da assistência.

\section{REFERÊNCIAS}

1. ABRAHÃO ALCL. A Unidade de Terapia Intensiva. In: CHEREGATTI AL, AMORIM CP (Org.). Enfermagem em unidade de terapia intensiva. 2. ed. São Paulo: Martinari, 2011; p. 17-39.

2. ABRGG PTGM, SILVA LL. Controle de infecção hospitalar em unidade de terapia intensiva: estudo retrospectivo. Semina: Ciências Biológicas e da Saúde, Londrina, jan./jun. 2011; 32(1): 47-58.

3. BACKES MTS, et al. O ambiente vivo, dinâmico e complexo de cuidados em Unidade de Terapia Intensiva. Rev. Latino-Am. Enfermagem, 2015; 23(3): 411-8.

4. BRASIL. Agência Nacional de Vigilância Sanitária. Corrente sanguínea. Critérios nacionais de infecções relacionadas à assistência à saúde, 2009.

5. BRASIL. Agência Nacional de Vigilância Sanitária. Indicadores Nacionais de Infecções Relacionadas à Assistência à Saúde, 2010.

6. BRASIL. Agência Nacional de Vigilância Sanitária. Medidas de prevenção de infecção relacionada à assistência à saúde [Internet]. Brasília: ANVISA, 2013.

7. BRASIL. Portaria n. 2616 de 12 de maio de 1998. Ministério da Saúde. Diário Oficial da União, Brasília, 12 de maio de 1998.

8. CALDANA G, et al. Indicadores de Desempenho em Serviço de Enfermagem Hospitalar: revisão integrativa. Rev. Rene, 2011; 12(1):189-97.

9. CONSELHO FEDERAL DE ENFERMAGEM. Resolução COFEN no 564/2017. Brasília, 2017.

10. FERNANDES ACL, et al. Sistematização da assistência de enfermagem na prevenção de infecções em unidade de terapia intensiva. Universidade Federal do Estado do Rio de Janeiro. Rio de Janeiro, Brasil Revista de Pesquisa Cuidado é Fundamental Online, 2014; 6(4): 1580-1589.

11. FIGUEIREDO DA. Fatores de Risco Associados à Infecção Hospitalar em uma Unidade de Terapia Intensiva. Dissertação (Mestrado) - UFPB/CCEN. João Pessoa, 2012.

12. FIGUEIREDO DA, et al. Epidemiologia da Infecção Hospitalar em uma Unidade de Terapia Intensiva de um Hospital Público Municipal de João Pessoa-PB. Revista Brasileira Ciência da Saúde, 2013; 17(3): 233-240.

13. FRANÇA FR, et al. Incidência de infecção relacionada à assistência à saúde na unidade de terapia intensiva de um hospital de médio porte. Revista Funec Científica - Multidisciplinar, 2020; 9(11). 
14. FRANÇA LM. A Gestão da qualidade associada ao controle de infecção em unidade de terapia intensiva. Revista Inspirar gestão e desenvolvimento, 2019 2; 3(1).

15. GARCIA PC, FUGULIN FMT. Tempo de assistência de enfermagem em unidade de terapia intensiva adulto e indicadores de qualidade assistencial: análise correlacional. Rev. Latino-Am. Enfermagem, 2012; 20(4).

16. GIROTI ALB, et al. Programas de Controle de Infecção Hospitalar: avaliação de indicadores de estrutura e processo. Rev. Escola de Enfermagem - USP, 2018; 1-7.

17. MAINZ J. Developing evidence-based clinical indicators: a state of the art methods primer. Int $\mathrm{J}$ Qual in Heal Care. 2003; 15 (Suppl 1): i5-i11.

18. MACHADO EA. Enfermagem em Unidade de Terapia Intensiva. Goiânia: AB, 2004; 168p.

19. MENEGUETI MG, et al. Infecção urinária em unidade de terapia intensiva: um indicador de processo para prevenção. Rev Rene, 2012; 13(3):632-8.

20. MENDES NR, et al. Sistematização da assistência de enfermagem e os desafios para sua implantação na unidade de terapia intensiva: uma revisão de literatura. Revista Uningá, 2019; 56(S2): 80-93.

21. PRATES DB, et al. Impacto de programa multidisciplinar para redução das densidades de incidência de infecção associada à assistência na UTI de hospital terciário em Belo Horizonte. Rev Med Minas Gerais, $2014 ; 66$ - 71.

22. RECH NLM, et al. Infecções primárias da corrente sanguínea em unidade de terapia intensiva do hospital de clínicas de Porto Alegre em 2018. 30. Semana de Enfermagem; promoção e realização Grupo de Enfermagem do Hospital de Clínicas de Porto Alegre, Escola de Enfermagem da Universidade Federal do Rio Grande do Sul; coordenação geral: Maria Luzia Chollopetz da Cunha. - Porto Alegre: HCPA, UFRGS, Escola de Enfermagem, 2019.

23. REIS ML, et al. Desigualdades sociais e a sustentabilidade da Amazônia. 2011.

24. REINER GL, et al. Desfecho clínico e fatores associados ao óbito em pacientes com sepse internados em unidade de terapia intensiva. Arquivos Catarinenses de Medicina, 2020; p. 2 - 9.

25. RODRIGES CN, PEREIRA DC. A. Infecções relacionadas à assistência à saúde ocorridas em uma Unidade de Terapia Intensiva. Rev. Investig. Bioméd., 2016; 8:41-51.

26. SANTANA TCP, et al. Dificuldades dos enfermeiros no atendimento ao pré-natal de risco habitual e seu impacto no indicador de morbimortalidade materno-neonatal. Revista Eletrônica Acervo Saúde (REAS), 2019, (20), e711.

27. SILVEIRA JS. Os Indicadores de Infecção Hospitalar e a Educação Permanente em Saúde: Uma Análise da Educação na redução dos Indicadores de Infecção Primária Relacionada ao Cateter Venoso Central. Trabalho de conclusão de curso (Especialização) - Universidade Federal do Rio Grande do Sul, Escola de Administração, Gestão em Saúde. Porto Alegre, BR-RS, 2019; p. 1-82

28. SALOMÉ M, et al. A comunicação durante a assistência ao paciente entubado internado em Unidade de Terapia Intensiva: a vivência dos alunos de graduação em enfermagem. Saúde Coletiva. Barueri, 2010; 37(7), p. 15-19.

29. VIEIRA MAN, BERETTA ALRZ. A eficácia de controle de infecção relacionada a assistência à saúde: revisão de literatura. Revista Científica FHO/ UNIARARAS, 2018; 6(2).

30. ZANARDO GM, et al. Sistematização da assistência de enfermagem. Revista Contexto \& Saúde, 2011; 10(20), 13711374. 